\title{
Use of Chemical Modification To Elucidate RNA Folding Pathways
}

Chemical modification reagents that react at the hydrogen-bonding groups of nucleotides, such as $\beta$-ethoxy- $\alpha$-ketobutyraldehyde (kethoxal), dimethyl sulfate (DMS), and 1-cyclohexyl-3-(2-morpholinoethyl)carbodiimide metho- $p$-toluenesulfonate (CMCT), react at nucleotides that are either single stranded or are at the end of helices (see UNIT 6.1; Inuoe and Cech, 1985; Moazed et al., 1986; Ehresmann et al., 1987). However, not all nucleotides that are not in Watson-Crick pairs are modified, because these reagents act based on the nucleotide's accessibility to solvent, which is sensitive to both secondary and tertiary structure (Banerjee et al., 1993; Mathews et al., 1997). Under pseudo-first-order reaction conditions (i.e., reagent concentration is much greater than RNA concentration), a linear relationship exists between rate of nucleotide modification and the fraction of accessible nucleotides; therefore, chemical modification can be used to measure the kinetics of RNA folding (Banerjee and Turner, 1995). This unit discusses the use of chemical modification reagents to measure RNA folding kinetics.

\section{CHOOSING A TRANSITION}

The first step in understanding the folding pathways of an RNA molecule is to identify the transitions of interest. A good starting point is to follow UV absorbance to identify transitions. RNA structure influences the extinction coefficient for the base transitions at 260 and 280 $\mathrm{nm}$ (Tinoco, 1959). For example, formation of secondary structure decreases the extinction coefficient. This phenomenon is often referred to as hypochromicity. Circular dichorism (CD) is an optical measurement that is even more sensitive to structural changes (Pan and Sosnick, 1997; UNIT 11.5). CD spectroscopy measures the difference in extinction coefficient for left and right circularly polarized light.

The extinction coefficient and CD spectrum can be measured for the RNA as a function of the denaturant that will be used to induce the transition. For example, an optical melting experiment is used if structural transitions are to be induced by changes in the temperature of the sample. Alternatively, a titration curve of extinction coefficient and/or CD as a function of $\mathrm{Mg}^{2+}$ concentration could be determined if an adjustment in $\mathrm{Mg}^{2+}$ concentration will be used to affect the transition of interest. The structural transitions are revealed by changes of extinc-



Contributed by David H. Mathews and Douglas H. Turner

RNA Folding Pathways

Current Protocols in Nucleic Acid Chemistry (2002) 11.9.1-11.9.4

11.9.1

Copyright $\odot 2002$ by John Wiley \& Sons, Inc. 
tion coefficient and/or CD. Structured RNAs typically have at least two transitions in optical melting curves (Crothers et al., 1974; Hilbers et al., 1976; Banerjee et al., 1993; Jaeger et al., 1993; Mathews et al., 1997). For example, Figure 11.9.1 shows the melting curve and its first derivative for the Tetrahymena thermophila group I ribozyme (Banerjee et al., 1993). The derivative reveals at least two transitions, one centered at $\sim 52^{\circ} \mathrm{C}$ and the other at $64^{\circ} \mathrm{C}$.

\section{CHARACTERIZING THE TRANSITION}

Once the transitions are identified, the structure should be mapped at equilibrium with chemical reagents at conditions both above and below the transitions (see UNIT 6.1). Consider the map of nucleotide accessibility to kethoxal in the T. thermophila group I ribozyme shown in Figure 11.9.2 (based on data from Banerjee et al., 1993). The structural transition centered at $52^{\circ} \mathrm{C}$ involves solvent exposure of many nucleotides, and is consistent with disruption of tertiary structure but retention of most secondary structure. For example, nucleotides G73, $\mathrm{G} 264$, and $\mathrm{G} 382$ are strongly modified at $65^{\circ} \mathrm{C}$, but not extensively modified at $50^{\circ} \mathrm{C}$.

Before determining folding kinetics, it is also advisable to test the effects of proposed perturbations on activity. For example, if folding will be initiated by lowering the temperature, it is useful to know if this perturbation results in fully functional RNA.
Use of Chemical Modification to Elucidate RNA Folding Pathways

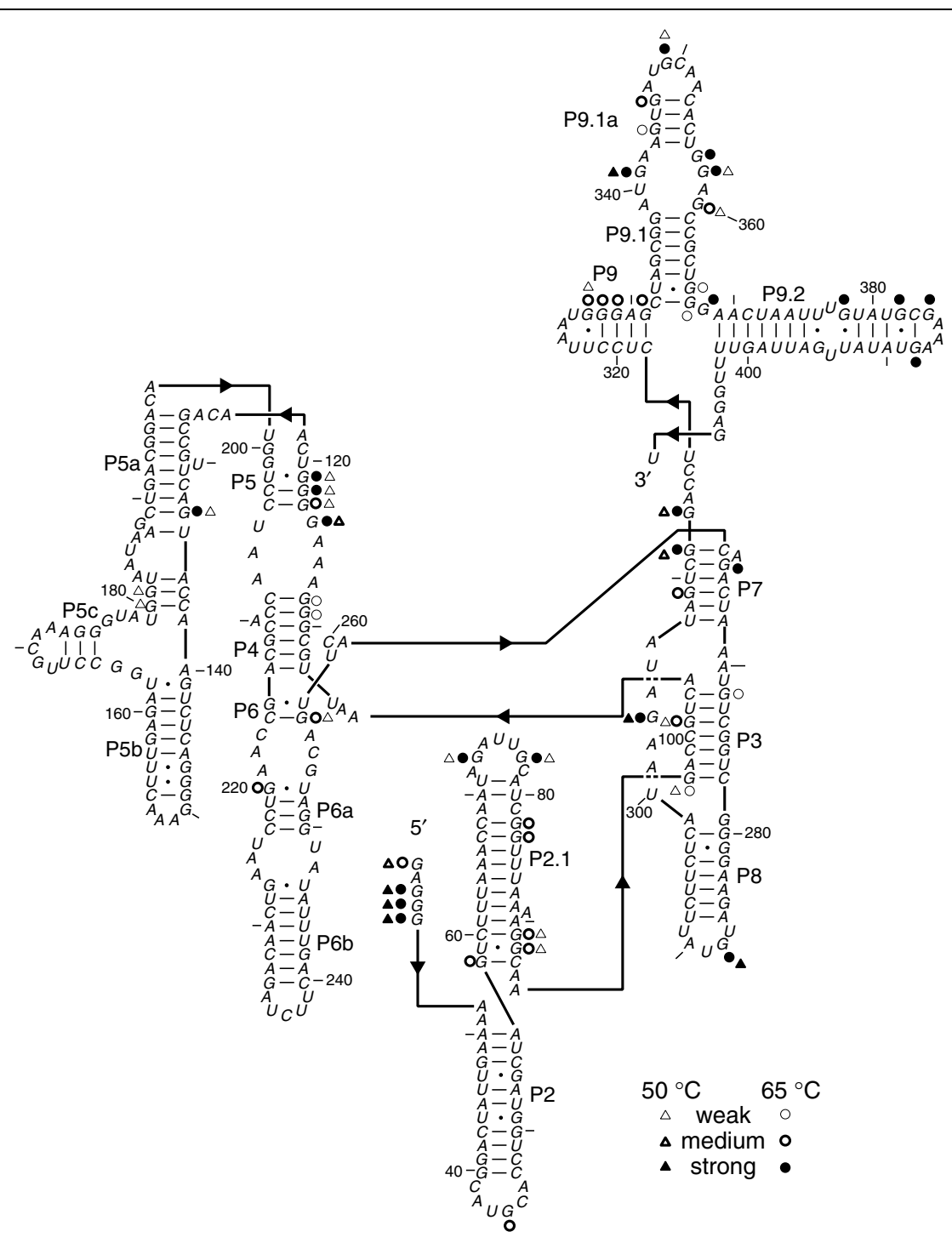

Figure 11.9.2 The map of kethoxal-accessible nucleotides at $50^{\circ}$ (triangles) and $65^{\circ} \mathrm{C}$ (circles). The modification intensity is quantified as weak, medium, and strong. 


\section{DETERMINING FOLDING \\ KINETICS}

To determine the kinetics of changing accessibility, equilibrate the RNA in a state of interest and then apply a structural perturbation along with modification reagent. As the structure reequilibrates, remove aliquots of modified RNA at a series of time points and quench the modification reaction. The time points are chosen to cover the time scale of folding under consideration. The extent of reaction as a function of time is later quantified by reverse transcription.

When interpreting the kinetics, the reactions of interest are:

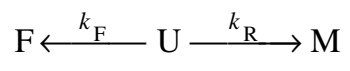

where each nucleotide is folded $(\mathrm{F})$, unfolded $(\mathrm{U})$, or modified (M). Only unfolded nucleotides are accessible to modification. Two rate constants apply: $k_{\mathrm{F}}$, the rate of folding, and $k_{\mathrm{R}}$, the rate of reaction with modification reagent. This scheme assumes that the folding of the nucleotide is first order and that the concentration of the modification reagent is much larger than the concentration of nucleotides, so that modification is pseudo first order. The fraction $\left(f_{t}\right)$ of modified nucleotides at time $t$ after exposure to the reagent will be:

$$
f_{t}=\left[\frac{k_{\mathrm{R}}}{k_{\mathrm{R}}+k_{\mathrm{F}}}\right]\left[1-\mathrm{e}^{-k_{F}^{t}}+k_{R}^{t e^{-k_{F} t}}\right]+f_{0}
$$

where $f_{0}$ is the apparent background modification that is a result of spontaneous strand cleavage and inefficiencies of reverse transcription (Banerjee and Turner, 1995). For nucleotides that do not become unreactive with time, $k_{\mathrm{F}}=$ 0 and this equation reduces to:

$$
f_{t}=k_{\mathrm{R}} t+f_{0}
$$

The concentration of reagent should be chosen such that the number of modifications per RNA molecule is less than one, so that $k_{\mathrm{R}} t<<$ 1. Then, for nucleotides that become protected with time, the fraction modified will be:

$$
f_{t}=\left[\frac{k_{\mathrm{R}}}{k_{\mathrm{R}}+k_{\mathrm{F}}}\right]\left[1-\mathrm{e}^{-k_{\mathrm{F}} t}\right]+f_{0}
$$

The fraction of modified nucleotide as a function of time can be fit to these equations to determine the rate constants.

For example, Banerjee and Turner (1995) studied the tertiary folding of the T. thermophila group I ribozyme from equilibrium at $60^{\circ} \mathrm{C}$ to equilibrium at $15^{\circ} \mathrm{C}$. The RNA was preequilibrated in $1 \mathrm{mM} \mathrm{Mg}^{2+}$ buffer at $60^{\circ} \mathrm{C}$ and then transferred to a larger volume of 10 $\mathrm{mM} \mathrm{Mg}{ }^{2+}$ buffer containing kethoxal at $15^{\circ} \mathrm{C}$. Modification was quantified at $5 \mathrm{sec}$ and 10 , $20,40,60,120,180,240,360$, and $480 \mathrm{~min}$ to determine folding rate constants from 0.048 to $0.008 \mathrm{~min}^{-1}$. The $5 \mathrm{sec}$ time point was used to determine $f_{0}$. Modification was visualized by reverse transcription of the RNA with labeled primer and the extent of modification was quantified with a phosphorimager.

On the basis of the tertiary folding time scales, the guanines shown in Figure 11.9.2 were placed into four classes: (1) guanines already protected or that fold with half-lives of less than $10 \mathrm{~min}$, (2) guanines that fold with time scales of tens of minutes, (3) guanines that continue to fold after an hour, and (4) guanines that never fold (Banerjee and Turner, 1995). Guanines that fold immediately include G44, G73, G341, G358, G360, G368, G382, and G384. These are clustered in loops at the $5^{\prime}$ and $3^{\prime}$ ends of the molecule, suggesting that the fast folding may involve interactions between the ends of the molecule.

Guanines that do not fold demonstrate a linear increase in the fraction modified as a function of time (third equation). Such guanines are typically single stranded or at the ends of helices.

\section{CONCLUSION}

Chemical modification is sensitive to solvent accessibility and can therefore be used to determine folding pathways. Measurements at equilibrium can reveal conditions inducing transitions. Measurements after a suitable perturbation can be used to determine the rates of these transitions at nucleotide resolution. Coupled with other methods, such as stopped-flow mixing (Bevilacqua et al., 1992), hydroxyl radical cleavage (Sclavi et al., 1998; Chaulk and MacMillan, 2000; Kent et al., 2000; UNIT 11.6), and oligonucleotide hybridization (Zarrinkar and Williamson, 1994), chemical modification can help provide a picture of the steps involved in folding a full-length RNA. It is quite possible, however, that the folding pathway for a full-length RNA will differ from that of the same RNA during transcription.
RNA Folding Pathways

11.9.3

Supplement 9 


\section{LITERATURE CITED}

Banerjee, A.R. and Turner, D.H. 1995. The time dependence of chemical modification reveals slow steps in the folding of a group I ribozyme. Biochemistry 34:6504-6512.

Banerjee, A.R., Jaeger, J.A., and Turner, D.H. 1993. Thermal unfolding of a group I ribozyme: The low temperature transition is primarily disruption of tertiary structure. Biochemistry 32:153163.

Bevilacqua, P.C., Kierzek, R., Johnson, K.A., and Turner, D.H. 1992. Dynamics of ribozyme binding of substrate revealed by fluorescence-detected stopped-flow methods. Science 258:13551358.

Chaulk, S.G. and MacMillan, A.M. 2000. Characterization of the Tetrahymena ribozyme folding pathway using the kinetic footprinting reagent peroxynitrous acid. Biochemistry 39:2-8.

Crothers, D.M., Cole, P.E., Hilbers, C.W., and Schulman, R.G. 1974. The molecular mechanism of thermal unfolding of Escherichia coli formylmethionine transfer RNA. J. Mol. Biol. 87:63-88.

Ehresmann, C., Baudin, F., Mougel, M., Romby, P., Ebel, J., and Ehresmann, B. 1987. Probing the structure of RNAs in solution. Nucl. Acids Res. 15:9109-9128.

Hilbers, C.W., Robillard, G.T., Shulman, R.G., Blake, R.D., Webb, P.K., Fresco, R., and Riesner, D. 1976. Thermal unfolding of yeast glycine transfer RNA. Biochemistry 15:1874-1882.

Inuoe, T. and Cech, T.R. 1985. Secondary structure of the circular form of the Tetrahymena rRNA intervening sequence: A technique for RNA structure analysis using chemical probes and reverse transcriptase. Proc. Natl. Acad. Sci. U.S.A. 82:648-652.

Jaeger, L., Westhof, E., and Michel, F. 1993. Monitoring of cooperative unfolding of the sunY group I intron of bacteriophage T4. J. Mol. Biol. 234:331-346.
Kent, O., Chaulk, S.G., and MacMillan, A.M. 2000. Kinetic analysis of the M1 RNA folding pathway. J. Mol. Biol. 304:699-705.

Mathews, D.H., Banerjee, A.R., Luan, D.D., Eickbush, T.H., and Turner, D.H. 1997. Secondary structure model of the RNA recognized by the reverse transcriptase from the $\mathrm{R} 2$ retrotransposable element. RNA 3:1-16.

Moazed, D., Stern, S., and Noller, H.F. 1986. Rapid chemical probing of conformation in $16 \mathrm{~S}$ ribosomal RNA and 30S ribosomal subunits using primer extension. J. Mol. Biol. 187:399-416.

Pan, T. and Sosnick, T.R. 1997. Intermediates and kinetic traps in the folding of a large ribozyme revealed by circular dichroism and UV absorbance spectroscopies and catalytic activity. Nat. Struct. Biol. 4:931-938.

Sclavi, B., Sullivan, M., Chance, M.R., Brenowitz, M., and Woodson, S.A. 1998. RNA folding at millisecond intervals by synchrotron hydroxyl radical footprinting. Science 279:1940-1943.

Tinoco, I. Jr. 1959. Hypochromism in polynucleotides. J. Am. Chem. Soc. 82:4785-4790.

Zarrinkar, P.P. and Williamson, J.R. 1994. Kinetic intermediates in RNA folding. Science 265:918924.

\section{KEY REFERENCES}

Banerjee and Turner, 1995. See above.

Presents the kinetics of folding of the T. thermophila group I ribozyme as determined by chemical modification with kethoxal.

Ehresmann et al., 1987. See above.

Reviews the activity and use of many popular chemical modification reagents.

Contributed by David H. Mathews and Douglas H. Turner

University of Rochester

Rochester, New York
Use of Chemical Modification to Elucidate RNA Folding Pathways

11.9.4

Supplement 9 